\title{
ORIGINAL
}

\section{MEDICIÓN DE LA GRASA CORPORAL MEDIANTE IMPEDANCIA BIOELÉCTRICA, PLIEGUES CUTÁNEOS Y ECUACIONES A PARTIR DE MEDIDAS ANTROPOMÉTRICAS. ANÁLISIS COMPARATIVO}

\author{
Vicente Martín Moreno, Juan Benito Gómez Gandoy y María Jesús Antoranz González
}

Centro de Salud Coronel de Palma. Móstoles. Madrid.

\section{RESUMEN}

Fundamento: La estimación del porcentaje de grasa corporal $(\% \mathrm{GC})$ está adquiriendo una importancia creciente a nivel clínico. El objetivo del estudio es comparar los resultados de $\% \mathrm{GC}$ obtenidos mediante técnicas aplicables en la consulta diaria: pliegues cutáneos, ecuaciones antropométricas e impedancia bioeléctrica (IB).

Métodos: Estudio descriptivo transversal. Participaron 149 personas, 83 varones y 66 mujeres. Se determinó altura, peso, circunferencia de la cintura (CC), pliegues cutáneos de bíceps, tríceps (PCT), subescapular y suprailíaco, suma y logaritmo de la suma de los cuatro pliegues. Se determinó el \%GC mediante las ecuaciones de Siri (global por sexo y específica por edad-sexo), Brozeck (global y específica), Deurenberg y Lean (para CC y CC-PCT) y por IB (Omron BF $300 \AA)$. La concordancia entre métodos se valoró mediante los coeficientes de correlación intraclase (CCI) y de Spearman (CCS) y el método de Bland-Altman. El método de referencia fue la ecuación de Siri-específica.

Resultados: Las medias de \%GC fueron $26,8 \pm 8,3 \%$ (Siri-específica), $25,7 \pm 8,1 \%$ (Siri-global), 25,97 \pm 7,6\% (Brozeck-específica), 24,9 \pm 7,5\% (Brozeck-global), 25,6 \pm $8,8 \%$ (IB), $28,6 \pm 8,2 \%$ (Deurenberg), $29,7 \pm 8,2 \%$ (Lean-cintura) y $29,4 \pm 9,3 \%$ (Lean-tríceps-cintura). Todos los métodos presentaron CCS $>0,88$ y CCI $>0,85$ con la ecuación Siri-específica. El mayor grado de acuerdo (Bland-Altman) lo presentaron las relaciones Siri-Brozeck específicas (media 0,8\%; intervalo $-0,48$ a $2,08 \%)$ y Siri-específica - IB $(1,17 ;-6,21$ a $8,55 \%)$

Conclusiones: Las ecuaciones de Deurenberg y Lean no son intercambiables con la IB y los pliegues cutáneos. Se propone la utilización de las ecuaciones de Siri-Brozeck para valorar el \%GC, sugiriéndose que el monitor Omron BF $300{ }^{\circledR}$ puede ser una alternativa válida.

Palabras clave: Composición corporal. Impedancia bioeléctrica. Grasa corporal. Antropometría. Pliegues cutáneos.

Correspondencia:

Vicente Martín Moreno

Calle del Alerce $5,5^{\circ} \mathrm{B}$

28041 Madrid

\section{ABSTRACT}

\section{Body Fat Estimated by Bioelectrical Impedance, Skinfold Thickness and Anthropometric Equations. A Comparative Analysis}

Background: There is growing clinical interest in estimating body fat percentages $(\% \mathrm{FM})$. The aim of this study was a comparison of body fat measurement by anthropometric equations, skinfold thickness (SFT) and bioelectrical impedance (BIA) methods

Methods: Cross-sectional study. 149 healthy individuals (83 males and 66 females) were recruited. Height, weight, waist circumference, skinfold of biceps, triceps, subscapular and suprailiac regions, sum and $\log _{10}$ sum of four folds were obtained. $\% \mathrm{FM}$ were calculated using the Siri equation (for sex or age-sex), Brozeck (sex or age-sex), Deurenberg, Lean and segmental BIA (Omron BF 300®). Methodological differences among the various methods were analyzed with Spearman (SCC) and intraclass (ICC) correlation coefficients and Bland-Altman method. The reference method is the Siri-age-sex equation.

Results: The means of \%FM were $26.8 \pm 8.3 \%$ (Siri-age-sex), $25.7 \pm 8.1 \%$ (Siri-sex), $25.97 \pm 7.6 \%$ (Brozeck-age-sex), $24.9 \pm 7.5 \%$ (Brozeck-sex), $25.6 \pm 8.8 \%$ (BIA), $28.6 \pm 8.2 \%$ (Deurenberg), $29.7 \pm 8.2 \%$ (Lean-waist circumference) and $29.4 \pm 9.3 \%$ (Lean-triceps-waist). The $\% \mathrm{FM}$ values for all methods were highly intercorrelated (all SCC $>0.83$ and CCI $>0.85$ ). Brozeck-age-sex (average difference $0.8 \%$; limits of agreement -0.48 to $2.08 \%)$ and BIA $(1.17 \% ;-6.21$ to $8.55 \%$ ) show the best agreement according to Bland and Altman analysis with Siri-age-sex equation.

Conclusions: Deurenberg and Lean equations provide different body fat mass estimates than those derived from SFT measurement and BIA and thus should not be used interchangeably. The use of Siri-Brozeck equations is recommended for $\% \mathrm{FM}$ assessment. The results suggest that segmental BIA-Omron BF $300 \AA$ may be a valid alternative method.

Key words: Body composition. Bioelectrical impedance analysis. Segmental BIA. Body fat. Anthropometry. Skinfold thickness. 


\section{INTRODUCCIÓN}

La aplicación de técnicas o medidas antropométricas para determinar la composición corporal, a excepción del peso, la talla y el índice de masa corporal (IMC), es poco utilizada en la práctica clínica diaria, reservándose fundamentalmente para estudios clínicos o epidemiológicos. En nuestro medio existen diversos estudios poblacionales realizados sobre la base de medidas antropométricas $^{1-6}$, pero son escasos los que toman como referencia las principales técnicas de valoración de la composición corporal: densitometría hidrostática (DH), absorciometría de rayos $\mathrm{X}$ de dos energías (DEXA) e impedancia bioeléctrica (IB). Este hecho contrasta con la creciente importancia que su determinación está adquiriendo tanto en el campo de la prevención, para detectar o valorar sujetos o poblaciones con aumento del riesgo de desarrollar enfermedades de alta prevalencia ${ }^{7-9}$ (hipertensión, diabetes, etc...), como en el del diagnóstico y seguimiento de estas y otras enfermedades: obesidad, anorexia, malnutrición ${ }^{7,10,11}$.

Entre las razones que justifican esta situación está la dificultad de acceso de los profesionales a la mayoría de estas técnicas ${ }^{12-14}$, técnicas que precisan la utilización de aparatos complejos, que requieren una gran infraestructura acompañante por su formato y/o sus características operativas y cuyo coste hace que queden reservadas a grandes centros hospitalarios, donde se utilizan generalmente para la realización de estudios en grupos, siendo la valoración individual poco frecuente. En el caso de las medidas antropométricas también influye que las ecuaciones en que se basan no han sido con frecuencia validadas para nuestra población, o bien, dentro de las que sí lo han sido o hay estudios $^{1-4,15}$, como es el caso de los pliegues cutáneos, porque requieren aparatos (plicómetro) que no siempre están disponibles en una consulta y entrenamiento previo.

Establecida la necesidad de determinar la grasa corporal, los requisitos que deben cumplir las técnicas seleccionadas deben ser: 1. que puedan ser aplicadas en la consulta diaria en razón de su accesibilidad (poder realizarse en el momento), sencillez de manejo, no invasibilidad, reproducibilidad y facilidad de ser aceptadas por el paciente como una técnica habitual de examen, tal como también proponen otros autores ${ }^{15} .2$. Que su coste pueda ser asumido por el sistema (coste de los aparatos necesarios) y el profesional (tiempo que lleva su realización). 3. Que exista evidencia suficiente de su utilidad para este fin ${ }^{12}$.

De todas las técnicas utilizadas en la valoración de la grasa corporal (DH, DEXA, tomografía, etc...), sólo la medición de los pliegues cutáneos, las ecuaciones basadas en medidas antropométricas y la IB cumplen estos requisitos ${ }^{16}$. Por este motivo el objetivo del presente estudio es realizar un análisis comparativo de los resultados obtenidos en la determinación de la grasa corporal en un grupo de personas sanas mediante las ecuaciones de $\operatorname{Siri}^{17}$ y Brozeck ${ }^{18}$, basadas exclusivamente en los pliegues cutáneos, las ecuaciones de Deurenberg ${ }^{19}$ y Lean $^{20}$, que incluyen otras medidas antropométricas además de los pliegues cutáneos, y la impedancia bioeléctrica, mediante el monitor personal OMRON BF 300®.

\section{MATERIAL Y MÉTODOS}

Estudio descriptivo transversal realizado en los centros de salud Coronel de Palma y San Fernando de Móstoles entre mayo y diciembre de 2000. Participan 149 personas aparentemente sanas, 83 varones y 66 mujeres, seleccionadas mediante petición de colaboración voluntaria entre la población que acude a consulta y el personal de los centros. Al desconocer la desviación estándar del porcentaje de grasa corporal $(\% \mathrm{GC})$ en nuestra población se tomó como referencia para el cálculo del tamaño de la muestra el estudio de Núñez y cols ${ }^{21}$, realizado sobre población de la misma comunidad autónoma, que aporta una desviación estándar de 
4,1\% para la ecuación de Siri, obteniendo, para una precisión de \pm una unidad en $\% \mathrm{GC}$ y un intervalo de confianza del $95 \%$, un tamaño muestral de 72. Para disminuir el error tipo II fueron finalmente incluidas 149 personas. Ninguna persona seleccionada rehusó participar.

Todas las medidas se realizaron en la misma sesión, para evitar que variaciones en las condiciones ambientales o biológicas afectaran a los resultados. Se realizaron primero las medidas antropométricas y después la medición por IB. Peso y talla se midieron estando el sujeto en ropa interior con una báscula-tallímetro Añó-Sayol®, con una precisión de 100 gramos (peso) y 1 $\mathrm{mm}$ (talla). El IMC se obtuvo mediante la relación $\mathrm{IMC}=$ peso $(\mathrm{kg}) /(\text { talla }(\mathrm{m}))^{2}$. Los pliegues cutáneos se midieron siguiendo los criterios de Durnin ${ }^{22}$ : 1. pliegue tricipital: longitudinalmente, en la parte posterior del miembro superior no dominante, en el punto medio entre acromion y olécranon, con la extremidad relajada, de forma paralela al eje del brazo; 2 . pliegue bicipital: en el mismo punto que el tricipital, pero en la cara anterior del brazo; 3 . pliegue subescapular: justo por debajo de la punta de la escápula no dominante, con un eje de $45^{\circ}$ respecto de la columna vertebral y 4. pliegue suprailíaco: por encima de la cresta ilíaca a nivel de la línea medio-axilar, formando un ángulo de $45^{\circ}$ con la línea inguinal media, con un plicómetro Levetta Project $\AA$ (precisión $0,5 \mathrm{~mm}$ ), tomando una superficie de pliegue de 6 por $11 \mathrm{~mm}$. Todas las mediciones se realizaron por triplicado por el mismo observador, con lectura a los cuatro segundos. La circunferencia de la cintura (CC) se midió en el momento de vacío entre el final de la espiración y el comienzo de la inspiración de una respiración normal, en el punto medio entre el margen costal inferior (borde inferior de la décima costilla) y la cresta ilíaca (espina ilíaca anterosuperior ${ }^{23}$, con una cinta métrica flexible MASS ${ }^{\circledR}$, tipo rollfix, de Hoechst ${ }^{\circledR}$, con precisión de $1 \mathrm{~mm}$.
Para la determinación del porcentaje de grasa corporal mediante IB se utilizó el monitor OMRON BF 300® (OMRON Matsukasa Co. LTD, Japón), que mide la impedancia de brazo a brazo a lo largo de la cintura escapular, es decir, en la parte superior del tronco. Todas las determinaciones se realizaron por triplicado, con un minuto de separación entre ellas, en ropa interior y posición de bipedestación, con las piernas separadas $35^{\circ}-45^{\circ}$ y los brazos extendidos hacia delante en ángulo recto $\left(90^{\circ}\right)$ respecto a la vertical del cuerpo, sin doblar los codos, con ayuno y sin haber realizado ejercicio en las tres horas previas.

Como ecuaciones para el cálculo del porcentaje de grasa corporal (\%GC) se seleccionaron las de Siri ${ }^{17}$, Brozeck ${ }^{18}$, Deurenberg ${ }^{19}$ y Lean ${ }^{20}$, que son las que cuentan con mayor aceptación. En las dos primeras la densidad corporal (D) se obtuvo mediante la ecuación propuesta por Durnin y Womersley $22: \mathrm{D}=\mathrm{C}-\mathrm{Mxlog}_{10} \Sigma$ cuatro pliegues, utilizando los coeficientes $\mathrm{C}$ y $\mathrm{M}$ de la suma de los cuatro pliegues reflejados en las tablas desarrolladas por estos autores, por un lado de forma global para cada sexo (densidad global) y por otro de forma específica para cada grupo de edad en cada sexo (densidad específica) (tabla 1).

La expresión matemática de la ecuación de Siri es:

$$
\begin{gathered}
\% \text { grasa corporal }= \\
=[(4,95 / \text { densidad })-4,5] \times 100
\end{gathered}
$$

y la de Brozeck:

$$
\begin{gathered}
\% \text { grasa corporal }= \\
=[(4,57 / \text { densidad })-4,142] \times 100
\end{gathered}
$$

Al utilizar la densidad global obtenemos las ecuaciones Siri-global y Brozeck-global y al utilizar la densidad específica las ecuaciones Siri-específica y Brozeck-específica. Aunque la Sociedad Española para el Estudio de la Obesidad (SEEDO) ${ }^{23}$ reconoce ambas formas de la ecuación de Siri como 
Tabla 1

Coeficientes C y M para la suma de los cuatro pliegues recogidos en las tablas de Durnin y Womersley ${ }^{22}$

\begin{tabular}{|c|c|c|c|c|c|c|c|}
\hline \multicolumn{8}{|c|}{ Varones } \\
\hline \multirow{3}{*}{$\begin{array}{c}\text { Suma de los } \\
\text { cuatro } \\
\text { pliegues }\end{array}$} & Edad $\rightarrow$ & $17-19$ & $20-29$ & $30-39$ & $40-49$ & $50+$ & $17-72$ \\
\hline & C & 1,1620 & 1,1631 & 1,1422 & 1,1620 & 1,1715 & 1,1765 \\
\hline & M & 0,0630 & 0,0632 & 0,0544 & 0,0700 & 0,0779 & 0,0744 \\
\hline \multicolumn{8}{|c|}{ Mujeres } \\
\hline \multirow{3}{*}{$\begin{array}{c}\text { Suma de los } \\
\text { cuatro } \\
\text { pliegues }\end{array}$} & Edad $\rightarrow$ & $16-19$ & $20-29$ & $30-39$ & $40-49$ & $50+$ & $16-68$ \\
\hline & C & 1,1549 & 1,1599 & 1,1423 & 1,1333 & 1,1339 & 1,1567 \\
\hline & M & 0,0678 & 0,0717 & 0,0632 & 0,0612 & 0,0645 & 0,0717 \\
\hline
\end{tabular}

técnica patrón para valorar el \%GC, en este estudio se utilizó la ecuación Siri-específica. Los efectos de utilizar la densidad global o específica se analizaron comparando entre sí ambas ecuaciones en función de la edad, factor diferenciador entre ellas, así como con relación al resultado obtenido con el monitor Omron BF $300 \AA$, al ser esta una técnica que no utiliza los pliegues cutáneos.

La ecuación de Deurenberg ${ }^{19}$ permite el cálculo del porcentaje de grasa corporal a partir del IMC y su expresión matemática para mayores de 15 años es:

$$
\begin{aligned}
& \% \text { de grasa corporal }=(1,2 \times \mathrm{IMC})+ \\
& +(0,23 \times \text { edad })-(10,8 \times \text { sexo })-5,4
\end{aligned}
$$

donde sexo $=1$ para varones y sexo $=0$ para mujeres. Lean, Han y Deurenberg, en un estudio posterior ${ }^{20}$, recogen diversas ecuaciones para el cálculo del \%GC a partir de diversas medidas antropométricas, reflejando que la que tiene mayor poder de predicción es la que se basa en la circunferencia de la cintura (CC) ajustada por edad, siendo su expresión para varones:

$$
\begin{gathered}
\% \text { grasa corporal }=(0,567 \times \mathrm{CC}-\mathrm{cm}-)+ \\
+(0,101 \times \text { edad })-31,8
\end{gathered}
$$

y para mujeres:

$$
\begin{gathered}
\% \text { grasa corporal }=(0,439 \times \mathrm{CC}-\mathrm{cm}-)+ \\
+(0,221 \times \text { edad })-9,4
\end{gathered}
$$

seguida, en cuanto a poder de predicción, de la ecuación que asocia la CC con el PT, cuyas expresiones son:

para varones

$$
\begin{gathered}
\% \text { grasa corporal }=(0,353 \times \mathrm{CC}-\mathrm{cm}-)+ \\
+(0,756 \times \mathrm{PT}-\mathrm{mm}-)+ \\
+(0,235 \times \text { edad }- \text { años- })-26,4
\end{gathered}
$$

y para mujeres

$$
\begin{gathered}
\% \text { grasa corporal }=(0,232 \times C \mathrm{C}-\mathrm{cm}-)+ \\
+(0,657 \times \mathrm{PT}-\mathrm{mm}-)+ \\
+(0,215 \times \text { edad -años- })-5,5
\end{gathered}
$$

El procesamiento y análisis de los datos se realizó de la siguiente forma:

1. expresando la media, desviación estándar y rango de las medidas antropométricas obtenidas, de forma global y por sexo.

2. Se determinó la distribución normal de la variable $\% \mathrm{GC}$ y de las diferencias entre métodos mediante la prueba de Kolmogorov-Smirnov con la corrección de Lilliefors y se comparó las medias de \%GC obtenidas por cada técnica mediante la prueba de la t de Student para datos apareados.

Para valorar la concordancia entre dos técnicas diferentes que miden la misma variable se utilizó el coeficiente de correlación intraclase (CCI), modelo de efectos aleato- 
$\operatorname{rios}^{24}$, y el método de Bland y Altman ${ }^{25}$. Cuando las diferencias entre las varianzas son significativas el CCI no es una medida válida del grado de acuerdo, por lo que en esta situación se calculó el coeficiente de correlación de Spearman, considerándose significativa toda $\mathrm{p} \leq 0,01$. En el resto de situaciones el límite de significación estadística se estableció en $\mathrm{p}<0,05$.

\section{RESULTADOS}

La media de edad de las personas que participaron en el estudio fue de $38,1 \pm 12$ años, con un intervalo de 16 a 62, tabla 2. Las diferencias en edad entre varones $(38,6 \pm 12,6$ años) y mujeres (37,4 $\pm 11,1$ años), así como en IMC $(26,7 \pm 3,8$ frente a $25,3 \pm$ $5,3)$, no fueron significativas. Las mujeres $(31,9 \pm 7,4 \%)$ presentan una mayor cantidad de grasa corporal que los varones $(22,7 \pm$ $6,5 \% ; \mathrm{p}<0,001)$.

El \%GC obtenido con la ecuación Siri-específica, técnica patrón, es de $26,8 \pm 8,3 \%$, mientras que con la ecuación Siri-global el resultado es de $25,7 \pm 8,1 \%$, diferencia que es significativa ( $<<0,001)$, tabla 3 . En la

Tabla 2

\begin{tabular}{|c|c|c|c|}
\hline \multicolumn{4}{|c|}{$\begin{array}{l}\text { Datos antropométricos de los sujetos incluidos en el estudio } \\
\text { Media } \pm \text { desviación estándar. Entre paréntesis, intervalo }\end{array}$} \\
\hline & Total & Varones & Mujeres \\
\hline Número de sujetos & 149 & 83 & 66 \\
\hline Edad (años) & $\begin{array}{l}38,1 \pm 12 \\
(16-62)\end{array}$ & $\begin{array}{l}38,6 \pm 12,6 \\
(17-62)\end{array}$ & $\begin{array}{l}37,5 \pm 11,1 \\
\quad(16-60)\end{array}$ \\
\hline Peso $(\mathrm{kg})$ & $\begin{array}{l}72,6 \pm 14,1 \\
(41-115,5)\end{array}$ & $\begin{array}{c}79,6 \pm 11,4 \\
(58,2-115,5)\end{array}$ & $\begin{array}{c}63,8 \pm 12,31 \\
(41-94,4)\end{array}$ \\
\hline Talla $(\mathrm{cm})$ & $\begin{array}{c}166,8 \pm 9,6 \\
(143,6-190)\end{array}$ & $\begin{array}{c}172,8 \pm 7,14 \\
(150-190)\end{array}$ & $\begin{array}{c}159,1 \pm 6,4 \\
(143,6-172)\end{array}$ \\
\hline IMC & $\begin{array}{c}26,1 \pm 4,5 \\
(15,5-38,6)\end{array}$ & $\begin{array}{c}26,7 \pm 3,8 \\
(19,1-38,5)\end{array}$ & $\begin{array}{c}25,3 \pm 5,3 \\
(15,5-38,6)\end{array}$ \\
\hline Circunferencia de la cintura $(\mathrm{cm})$ & $\begin{array}{l}88,9 \pm 13,9 \\
(59,6-127)\end{array}$ & $\begin{array}{l}95,1 \pm 10,9 \\
(73,6-127)\end{array}$ & $\begin{array}{c}81 \pm 13,4 \\
(59,6-124)\end{array}$ \\
\hline Pliegue del tríceps (mm) & $\begin{array}{l}15,6 \pm 7,2 \\
(2-32,3)\end{array}$ & $\begin{array}{l}12,1 \pm 5,6 \\
(2-31,2)\end{array}$ & $\begin{array}{c}19,98 \pm 6,6 \\
(7-32,3)\end{array}$ \\
\hline Pliegue del bíceps (mm) & $\begin{array}{c}7,4 \pm 5,3 \\
(0,5-29,8)\end{array}$ & $\begin{array}{c}5,7 \pm 3,9 \\
(0,5-21,7)\end{array}$ & $\begin{array}{c}9,6 \pm 6 \\
(2,3-29,8)\end{array}$ \\
\hline Pliegue subescapular (mm) & $\begin{array}{l}17,2 \pm 7,9 \\
(3,8-39,5)\end{array}$ & $\begin{array}{l}16,4 \pm 7,2 \\
(4,5-39,5)\end{array}$ & $\begin{array}{l}18,2 \pm 8,8 \\
(3,8-35)\end{array}$ \\
\hline Pliegue suprailíaco (mm) & $\begin{array}{l}19,3 \pm 7,7 \\
(2,5-36,2)\end{array}$ & $\begin{array}{l}20 \pm 7,5 \\
(3-36,2)\end{array}$ & $\begin{array}{c}18,4 \pm 8 \\
(2,5-31,2)\end{array}$ \\
\hline$\sum_{4}$ pliegues $(\mathrm{mm})$ & $\begin{array}{l}59,5 \pm 25,1 \\
(11-124,5)\end{array}$ & $\begin{array}{l}54,2 \pm 22,2 \\
(11-122,5)\end{array}$ & $\begin{array}{c}66,1 \pm 27 \\
(19,8-124,5)\end{array}$ \\
\hline $\log _{10} \sum_{4}$ pliegues $(\mathrm{mm})$ & $\begin{array}{c}1,7327 \pm 0,1997 \\
(1,04-2,10)\end{array}$ & $\begin{array}{c}1,6961 \pm 0,1916 \\
(1,04-2,09)\end{array}$ & $\begin{array}{c}1,7787 \pm 0,2017 \\
(1,30-2,10)\end{array}$ \\
\hline Siri-específica $(\% \mathrm{GC})$ & $\begin{array}{c}26,8 \pm 8,3 \\
(4,5-44,7)\end{array}$ & $\begin{array}{c}22,7 \pm 6,5 \\
(4,5-37,3)\end{array}$ & $\begin{array}{c}31,9 \pm 7,4 \\
(13,9-44,7)\end{array}$ \\
\hline
\end{tabular}


Tabla 3

Porcentaje de grasa corporal obtenido mediante impedancia bioeléctrica (IB), pliegues cutáneos y ecuaciones antropométricas Media \pm desviación estándar. Entre paréntesis, intervalo

\begin{tabular}{|lccc|}
\hline & Total & Varones & Mujeres \\
\hline Número de sujetos & 149 & 83 & 66 \\
\hline Siri-específica (\%GC) & $\begin{array}{c}26,8 \pm 8,3 \\
(4,5-44,7)\end{array}$ & $\begin{array}{c}22,7 \pm 6,5 \\
(4,5-37,3)\end{array}$ & $\begin{array}{c}31,9 \pm 7,4 \\
(13,9-44,7)\end{array}$ \\
\hline Siri-global (\%GC) & $\begin{array}{l}25,7 \pm 8,1 \\
(0,4-41,8)\end{array}$ & $\begin{array}{c}21,4 \pm 6,3 \\
(0,4-34,8)\end{array}$ & $\begin{array}{c}31,1 \pm 6,7 \\
(15,4-41,8)\end{array}$ \\
\hline Brozeck-específica (\%GC) & $\begin{array}{l}25,97 \pm 7,6 \\
(4,5-42,5)\end{array}$ & $\begin{array}{c}22,7 \pm 6,5 \\
(4,5-37,3)\end{array}$ & $\begin{array}{c}30,7 \pm 6,8 \\
(14,1-42,5)\end{array}$ \\
\hline Brozeck-global (\%GC) & $\begin{array}{c}24,9 \pm 7,5 \\
(1,6-39,9)\end{array}$ & $\begin{array}{c}21 \pm 5,9 \\
(1,6-33,3)\end{array}$ & $\begin{array}{c}29,9 \pm 6,2 \\
(15,4-39,9)\end{array}$ \\
\hline Deurenberg (\%GC) & $\begin{array}{c}28,6 \pm 8,2 \\
(11,6-52,7)\end{array}$ & $\begin{array}{c}24,7 \pm 6 \\
(11,6-42,4)\end{array}$ & $\begin{array}{c}33,6 \pm 7,9 \\
(18-52,7)\end{array}$ \\
\hline Lean para cintura (\%GC) & $\begin{array}{l}29,7 \pm 8,2 \\
(12-56,3)\end{array}$ & $\begin{array}{c}26 \pm 6,8 \\
(12-45,7)\end{array}$ & $\begin{array}{c}34,4 \pm 7,6 \\
(21,4-56,3)\end{array}$ \\
\hline Lean para tríceps y cintura (\%GC) & $\begin{array}{l}29,4 \pm 9,3 \\
(7,3-55,1)\end{array}$ & $\begin{array}{c}25,4 \pm 8,1 \\
(7,3-46,4)\end{array}$ & $\begin{array}{c}3 \\
(17,5 \pm 4-5,3\end{array}$ \\
\hline \%GC por IB Omron BF 300 & $\begin{array}{l}25,6 \pm 8,8 \\
(4,1-44,9)\end{array}$ & $\begin{array}{c}21,7 \pm 7,1 \\
(4,1-38,4)\end{array}$ & $\begin{array}{c}30,5 \pm 8,4 \\
(6,6-44,9)\end{array}$ \\
\hline
\end{tabular}

valoración del grado de acuerdo entre ambas ecuaciones, el coeficiente de correlación de Spearman fue 0,932 ( $<<0,001)$ (tabla 4), mientras que por el método de Bland y Altman la media de las diferencias es de $1,11 \%$ y el intervalo de concordancia se sitúa entre $-4,37$ y $6,59 \%$ (tabla 4 , figura 1 ).

Con la ecuación Brozeck-específica el $\%$ GC $(25,97 \pm 7,6 \%)$ es inferior al obtenido con la ecuación Siri-específica ( $\mathrm{p}<$ 0,001). El coeficiente de correlación de Spearman fue $1,000(p<0,001)$, presentando una media de las diferencias de $0,8 \%$ y un intervalo de concordancia entre $-0,48$ y $2,08 \%$, figura 2 . E igual ocurre con la ecuación Brozeck-global $(24,9 \pm 7,5 \%$; p < $0,001)$, situándose en este caso el coeficiente de correlación en $0,932(\mathrm{p}<0,001)$, con una media de las diferencias de $1,8 \%$ y un intervalo de concordancia de $-3,7$ a $7,3 \%$.
La ecuación de Deurenberg aporta un $\%$ GC más elevado $(28,6 \pm 8,2 \% ; \mathrm{p}<0,001)$ que la ecuación Siri-específica, presentando con ella un coeficiente de correlación de Spearman de 0,895 $(\mathrm{p}<0,001)$, una media de las diferencias de $-1,87 \%$ y un intervalo de concordancia entre $-9,07$ y 5,33 (figura 2).

La ecuación de Lean para cintura $(29,7 \pm$ $8,2 \% ; \mathrm{p}<0,001)$ ofrece un resultado de $\% \mathrm{GC}$ más elevado que el obtenido con la ecuación Siri-específica, con un coeficiente de correlación de Spearman de 0,894 (p < $0,001)$, una media de las diferencias de $-2,99 \%$ y un intervalo de concordancia entre $-10,39$ y $4,41 \%$, figura 2 . Esta diferencia se mantiene con la ecuación de Lean para pliegue del tríceps y cintura $(29,4 \pm 9,3 \%$; p $<0,001)$, que presenta un coeficiente de correlación de $0,939(\mathrm{p}<0,001)$, una media de las diferencias de $-2,66 \%$ y un intervalo de concordancia entre $-8,94$ y $3,62 \%$. 
MEDICIÓN DE LA GRASA CORPORAL MEDIANTE IMPEDANCIA BIOELÉCTRICA.

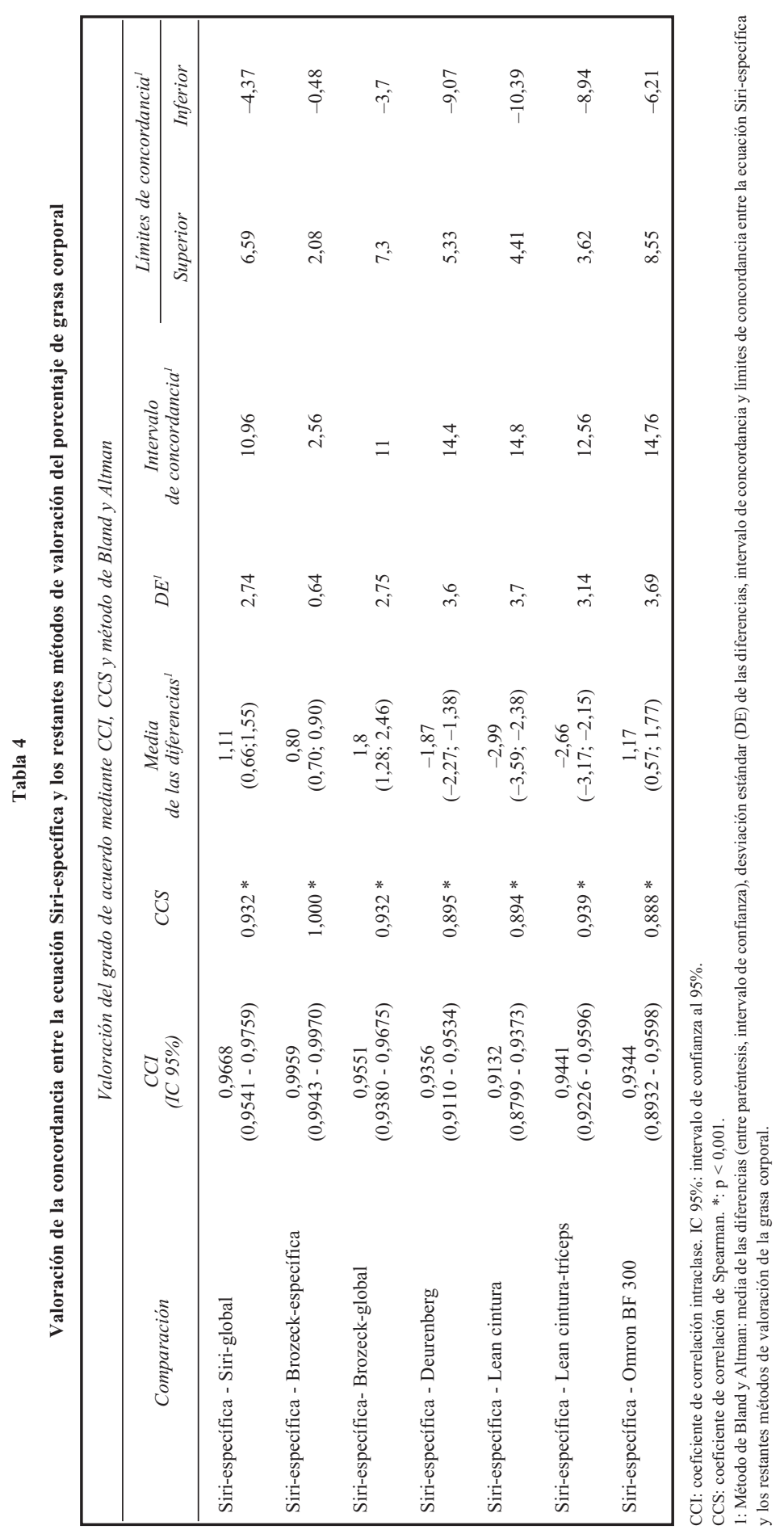

Rev Esp Salud Pública 2001, Vol. 75, N. ${ }^{\circ} 3$ 
Valoración de la concordancia entre ecuación Siri-específica y ecuación Siri-global, según el método de Bland y Altman. \%GC: porcentaje de grasa corporal

Valoración de la grasa corporal según el método de Bland y Altman

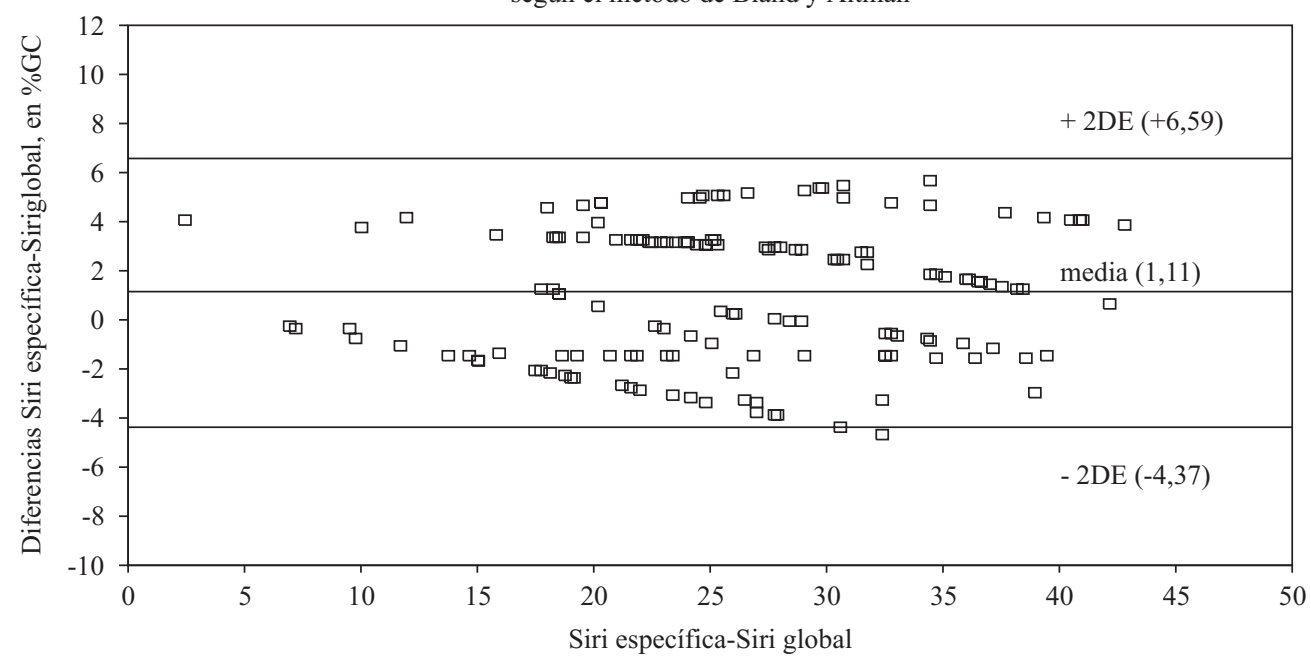

Media de cada caso entre los dos métodos, en \%GC

$\mathrm{El} \% \mathrm{GC}$ determinado por IB con el monitor Omron BF $300(25,6 \pm 8,8 \%)$ es inferior $(\mathrm{p}<0,001)$ al obtenido con la ecuación Siri-específica. El coeficiente de correlación de Spearman entre ambas técnicas es de $0,888(\mathrm{p}<0,001)$, con una media de las diferencias de $1,17 \%$ y un intervalo de concordancia entre $-6,21$ y $8,55 \%$.

Por último, además de la de Siri otras dos ecuaciones tienen dos variantes, la de Brozeck y la de Lean. La correlación entre las ecuaciones Brozeck-específica y Brozeck-global es $0,932$ ( $\mathrm{p}<0,001)$, mostrando una media de las diferencias de 1,02 $\pm 2,53 \%$ (intervalo de confianza al 95\% 0,61 a 1,43), un intervalo de concordancia entre $-4,03$ y $6,07 \%$ y una diferencia significativa entre sus medias (tabla 3 ). El CCI entre Lean para cintura y Lean para pliegue del tríceps y cintura es de 0,9707 , con IC al $95 \%$ por encima de 0,85 . La media de las diferencias es de $0,33 \pm 2,96 \%$ (intervalo de confianza al $95 \%-0,15$ a 0,81 ), con un intervalo de concordancia de $-5,59$ a $6,25 \%$. La diferencia entre sus medias, tabla 3 , no es significativa.

El menor \%GC se obtuvo con la ecuación Brozeck-global $(24,9 \pm 7,5 \%)$ y el más elevado con la ecuación de Lean para la cintura $(29,7 \pm 8,2 \%)$. La diferencia entre ambos es de $4,8 \%$. Salvo las diferencias Omron BF 300 - Siri-global, Omron BF 300 - Brozeck específica y global, Siri-global - Brozeck-específica y Lean cintura - Lean tríceps-cintura, todas las diferencias entre ecuaciones fueron significativas $(\mathrm{p}<0,001)$.

Con relación a la ecuación Siri-específica, las ecuaciones de Deurenberg, Lean-cintura y Lean-tríceps-cintura sobreestiman el \%GC, siendo la diferencia de $1,8 \%, 2,9 \%$ y $2,6 \%$ respectivamente, mientras que las ecuaciones Siri-global $\quad(-1,1 \%), \quad$ Brozeck-específica $(-0,8 \%)$, Brozeck-global $(-1,9 \%)$ y el monitor Omron BF $300(-1,2 \%)$ lo subestiman. Las ecuaciones Siri-global y Brozeck específica y global aportan un resultado similar al obtenido por IB con el monitor Omron BF 300. 


\section{Figura 2}

Valoración de la concordancia entre ecuación Siri-específica y ecuaciones Brozeck- específica, Brozeck-global, Deurenberg, Lean para cintura, Lean para tríceps y cintura y monitor por impedancia bioeléctrica Omron BF 300, según el método de Bland y Altman. \%GC: porcentaje de grasa corporal
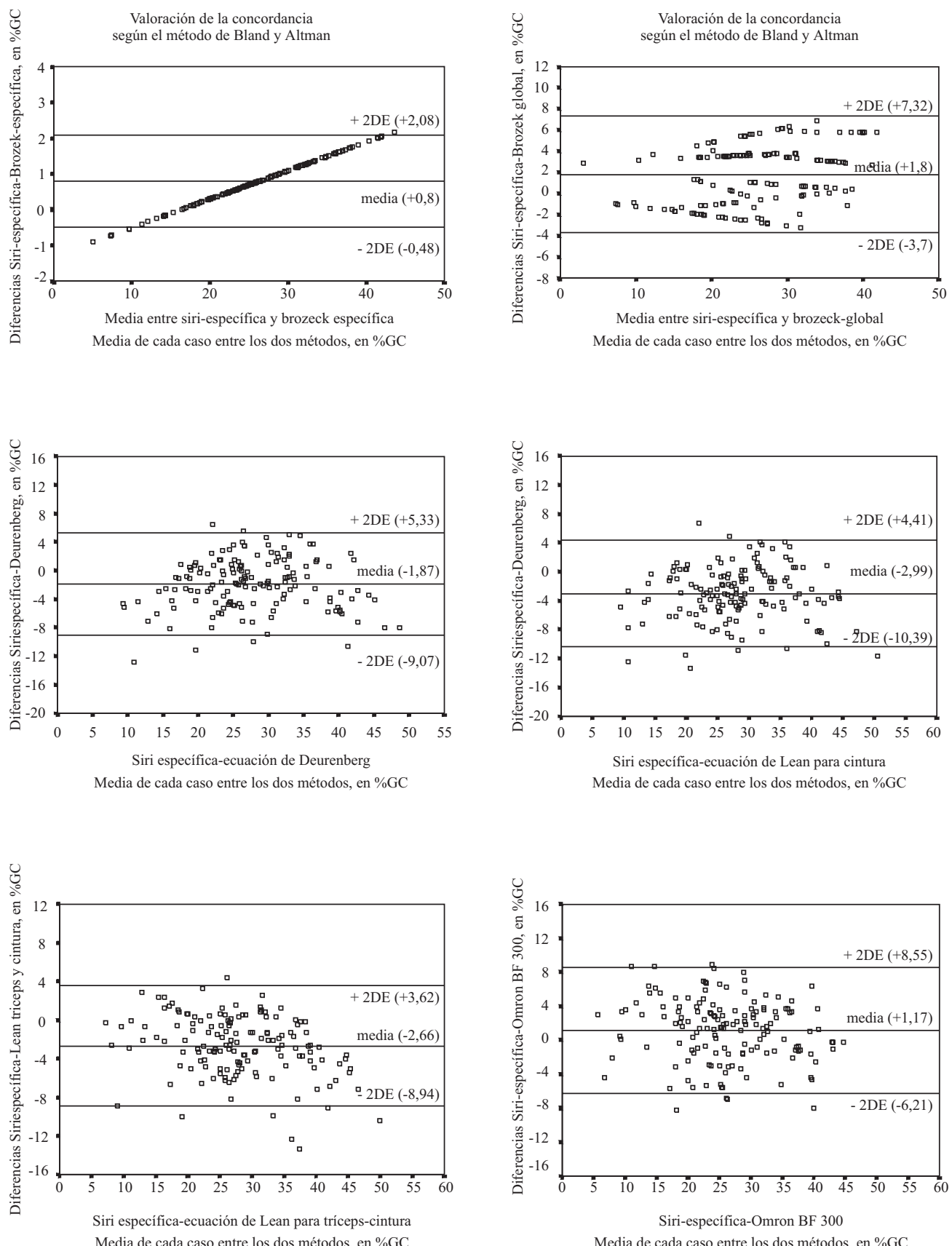

Rev Esp Salud Pública 2001, Vol. 75, N. ${ }^{\circ} 3$ 


\section{Figura 3}

Influencia en el resultado de \%GC de la utilización de la densidad específica o de la densidad global en las ecuaciones, en función de la edad y comparadas con el monitor Omron BF 300
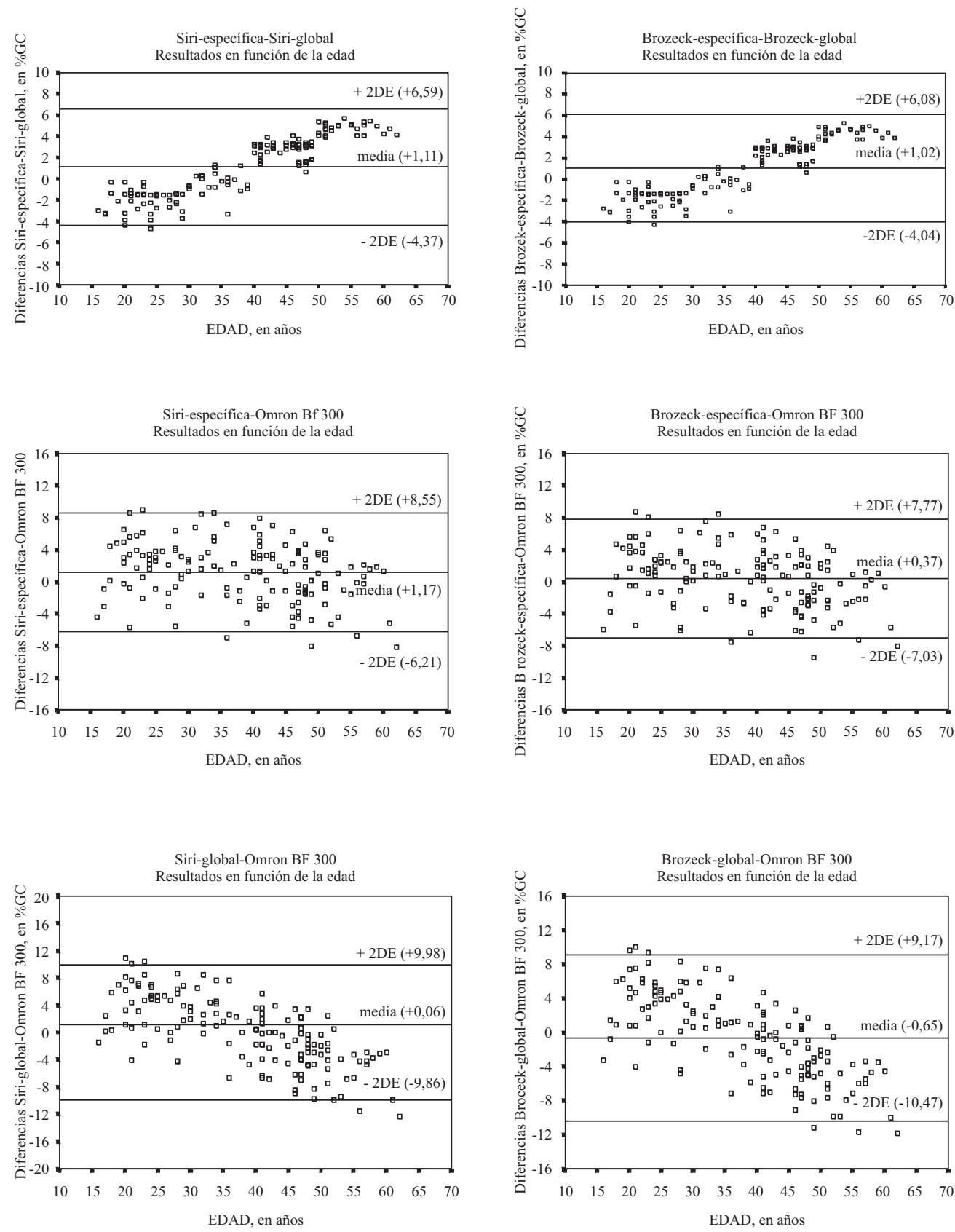
La relación observada entre el \%GC obtenido mediante las ecuaciones Siri-específica y Brozeck-específica es estrecha (coeficientes de correlación de Pearson y Spearman $\mathrm{r}=$ $1,000 ; \mathrm{p}<0,001)$, tanto en varones como en mujeres.

La utilización de la densidad global para el cálculo del \%GC en las ecuaciones de Siri y Brozeck condiciona que en sujetos menores de 40 años se produzca una subestimación del \%GC y por encima de los 40 años una sobrestimación con las ecuaciones Siri-global y Brozeck-global, figura 3. Este efecto se mantiene al comparar estas ecuaciones con otras técnicas, como la IB.

\section{DISCUSIÓN}

En este estudio se han aplicado a un grupo de sujetos sanos diversas técnicas y ecuaciones antropométricas que permiten estimar el $\%$ GC. Aunque el rango de IMC es amplio, no se incluyeron sujetos con obesidad mórbida (IMC $\geq 40$ ) por la dificultad de valorar adecuadamente en ellos los pliegues cutáneos $^{26-28}$ y la circunferencia de la cintura y ninguno de los sujetos con IMC $<20$ presentaba enfermedad subyacente. Los métodos analizados realizan una estimación indirecta del porcentaje de grasa corporal ${ }^{12-14,16}$, por lo que, en ausencia de un patrón oro como la densitometría, no es posible conocer cuál de ellos aporta una medida del \%GC más fiable. Los pliegues cutáneos, presentes en la mayoría de estudios sobre composición corporal como técnica de comparación con el método analizado y/o el de referencia, muestran una elevada concordancia o correlación con el $\% \mathrm{GC}$ obtenido a través de diversas técnicas: densitometría ${ }^{18-20,26,27,29,30}$, DEXA ${ }^{15,31}$, métodos dilucionales ${ }^{22,32}$ e IB $^{21}$, lo que, unido a su coste y accesibilidad, justifica su amplio uso en este tipo de estudios y que puedan ser considerados como el patrón oro entre las medidas antropométricas. La SEEDO recomienda la utilización de los pliegues cutáneos y la ecuación de Siri para la valoración del por- centaje de grasa corporal ${ }^{23}$, dando por válidas tanto la forma global como la forma específica de calcular la densidad corporal con la ecuación de Durnin-Womersley. Diversos artículos de revisión ${ }^{12,13,14,16,26,33}$ reflejan que la ecuación de Durnin y Womersley puede ser utilizada con seguridad en diversos grupos de población y, además, existen estudios en nuestro medio frente a técnicas de referencia ${ }^{15,21,31}$.

Las limitaciones del CCI y del coeficiente de correlación de Spearman en la valoración del grado de acuerdo entre técnicas ${ }^{24,34}$ aconsejaron la utilización complementaria del método de Bland y Altman, que permite valorar la importancia clínica de las diferencias observadas, tomando como referentes para esta valoración la amplitud del intervalo de concordancia, que representa a la media de las diferencias más-menos dos desviaciones estándar de las diferencias, y a la propia media de las diferencias, cuya mayor o menor separación de la diferencia nula, es decir, de cero, nos informa de la bondad del acuerdo. El CCI se situó en todos los casos por encima de 0,85 , incluido el límite inferior del intervalo de confianza al $95 \%$, por lo que el grado de concordancia es muy bue$\mathrm{no}^{34,35}$. Cuando las varianzas no fueron homogéneas se utilizó el coeficiente de correlación de Spearman, que fue siempre mayor de 0,88 .

En este estudio la diferencia de \%GC entre las ecuaciones Siri-específica y Siri-global es de un $1,1 \%$, en peso 799 gramos, con un intervalo de concordancia según el método de Bland y Altman de $10,9 \%$, diferencia e intervalo que, tomando como referencia los criterios de la SEEDO, se asumen como clínicamente aceptables, por lo que, dada la ausencia de normas de interpretación de los resultados establecidas por grupos de consenso, pueden ser utilizados como criterio de concordancia bueno (media de las diferencias $\leq 1,1 \%$, intervalo de concordancia $\pm 6,5 \%$ - el mayor de los dos límites de concordancia-) al comparar las diversas técnicas. También parece razonable desde el 
punto de vista clínico elevar este criterio de normalidad hasta una diferencia de peso de grasa corporal entre métodos de un kilogramo, que en función de la media de peso corporal obtenido representa un \%GC de $\pm 1,4 \%$ y que, como se refleja en otros estu$\operatorname{dios}^{20,32,36-39}$, los límites del intervalo de concordancia no superen el $\pm 10 \%$, estableciendo este segundo nivel como criterio de concordancia aceptable (media de las diferencias $>1,1 \%$ y $\leq 1,4 \%$, intervalo de concordancia con límites que no exceden el $\pm 10 \%$ ). Por fuera de estos valores las técnicas no serían intercambiables.

La ecuación Brozeck-específica presenta una correlación perfecta con la ecuación Siri-específica, método de referencia, y una buena concordancia desde el punto de vista clínico con el método de Bland-Altman, con el intervalo más estrecho de todas las comparaciones realizadas, por lo que ambos métodos son intercambiables.

Con la ecuación Brozeck-global la correlación sigue siendo estrecha, con un intervalo de concordancia que recibe la categoría de aceptable, pero la diferencia entre ambos métodos se sitúa fuera del rango del $\pm 1,4 \%$ y no puede considerarse una alternativa válida a la ecuación Siri-específica, ya que subestima el \%GC. Al comparar entre sí las dos variantes de la ecuación de Brozeck, específica y global, los resultados son similares a los obtenidos con la ecuación de Siri, tanto en diferencia de \%GC como en rango del intervalo de concordancia.

Al utilizar la densidad global en las ecuaciones de Siri y Brozeck se simplifica el cálculo del $\% \mathrm{GC}$, pero se pierde fiabilidad como consecuencia del desplazamiento hacia valores medios de los coeficientes $\mathrm{C}$ y $\mathrm{M}$ y aunque en este estudio este efecto sólo implica sobre el resultado final una mayor variabilidad, expresada a través de un mayor intervalo de concordancia con el método de Bland y Altman para las ecuaciones que utilizan la densidad global, en estudios sobre colectivos etarios más restringidos (jóvenes, mayores de 50 años) puede suponer un sesgo importante que debe ser tenido en cuenta. Por ello, parece oportuno recomendar la utilización de forma habitual de la densidad específica y no de la densidad global para el cálculo del \%GC.

Aunque el CCI y el coeficiente de correlación de Spearman entre la ecuación Siri-específica y las ecuaciones de Deurenberg, Lean para cintura y Lean para tríceps y cintura muestra un elevado grado de acuerdo, a nivel clínico se observa una importante variabilidad en los resultados, expresada con el método de Bland y Altman por un amplio intervalo de concordancia, alguno de cuyos límites sobrepasa el $\pm 10 \%$, y por una tendencia clara a ofrecer un $\% \mathrm{GC}$ más elevado, que queda reflejado en la media de las diferencias, que se aleja significativamente de cero y sobrepasa el $\pm 1,4 \%$ establecido como límite de acuerdo. Con ello, estas ecuaciones no son intercambiables con la ecuación Siri-específica para el cálculo del \%GC en nuestra población.

Las ecuaciones de Deurenberg y Lean ofrecen estimaciones del \%GC más elevadas que las obtenidas mediante IB y los pliegues cutáneos. Entre otros factores, las variaciones constitucionales o étnicas ${ }^{32,38,41}$ entre los grupos comparados han podido influir en este resultado. Así, la población participante en nuestro estudio presenta una talla media inferior, existiendo una diferencia de $8 \mathrm{~cm}$ respecto a los estudios de Lean y Deurenberg y de sólo $2 \mathrm{~cm}$ respecto a la de Durnin-Womersley, y un IMC mayor.

Estas variaciones podrían explicar las diferencias encontradas con la ecuación de Deurenberg, pero las ecuaciones de Lean y Durnin-Womersley (pliegues cutáneos) se han obtenido sobre población del mismo entorno (Glasgow), por lo que la diferencia de resultados entre ellas observada en nuestro estudio no debiera ser tan acusada. Al revisar el estudio de Lean ${ }^{20}$ encontramos que entre el \%GC obtenido mediante su ecuación y el calculado para la de Durnin-Womersley 
por este mismo autor existe una marcada diferencia $(\mathrm{p}<0,001)$, por lo que ambos métodos ofrecen ya desde su origen resultados diferentes y justifica la diferencia encontrada en nuestro estudio.

Con relación a la IB, la concordancia entre el \%GC obtenido con la ecuación Siri-específica y con el monitor Omron BF $300 \circledR$ es alta, con un CCI con límite inferior del intervalo de confianza al $95 \%$ por encima de 0,85 y un coeficiente de correlación de Spearman mayor de 0,88 . Con el método de Bland y Altman observamos que, aunque el monitor subestima el \%GC respecto a la técnica patrón, la media de las diferencias se sitúa dentro del margen establecido como aceptable, y también que a pesar de que el intervalo de concordancia es amplio, reflejo de una variabilidad importante entre los resultados ofrecidos por ambos métodos, se encuentra dentro de la categoría establecida como aceptable, por lo que, en concordancia con lo reflejado por otros estudios ${ }^{21,37}$, ambos métodos son intercambiables.

Como resumen, para determinar el \%GC en la consulta diaria disponemos de diversas herramientas, que permiten obtener una estimación del valor real. El método más utilizado es la ecuación de Siri, siendo recomendable usar la variante Siri-específica, ya que aporta un resultado más fiable al tener en cuenta en la valoración de la grasa corporal la influencia del sexo y la edad. La ecuación Brozeck-específica supone una buena alternativa a la ecuación Siri-específica en nuestra población, confirmando los resultados obtenidos en otras poblaciones ${ }^{12,13,21,40,41}$, aunque aporta pocas ventajas reales, ya que ambas se obtienen a partir de los mismos pliegues cutáneos y sólo varía la fórmula empleada para el cálculo, por lo que a nivel operativo el resultado queda limitado a que se puede utilizar una u otra para realizar un estudio.

En concordancia con los resultados obtenidos por Loy y cols. ${ }^{42}$ y Gibson y cols..$^{43}$ en grupos poblacionales de similares caracte- rísticas, el monitor por IB Omron BF $300 \AA$ también es una alternativa válida a la ecuación Siri-específica en la valoración de la grasa corporal a nivel clínico y epidemiológico, aportando como ventajas frente a los pliegues menor consumo de tiempo, inmediatez en el resultado, no ser molesto para el paciente, requerir mínimo entrenamiento y que la variabilidad intra e interobservador es menor ${ }^{12-14,16}$, aunque para su utilización es preciso conocer sus fundamentos y limitaciones $^{16,41,44}$. Sin embargo, otros estudios reflejan que este monitor subestima de forma importante el \%GC en personas jóvenes y delgadas ${ }^{45}$ y aunque en esta situación la capacidad de los pliegues para valorar el \%GC está limitada ${ }^{14,30,46}$, ya que el propio grosor de la piel puede suponer un sesgo nada despreciable de cara al resultado final, y la relación grasa subcutánea - grasa interna puede ser diferente, esta diferencia aconseja la realización de estudios complementarios con este monitor en otros colectivos (delgados, obesos, ancianos). El resto de ecuaciones, exceptuando la ecuación Siri-global, no son intercambiables con la ecuación Siri-específica, resultado que resalta la conveniencia de validar previamente las ecuaciones desarrolladas en otras poblaciones y la necesidad de contar con estudios específicos de nuestra población ${ }^{15,33}$. La importante variabilidad individual observada en este y otros estudios que comparan técnicas que se fundamentan en principios físicos o antropométricos diferentes (densitometría, DEXA, etc. $)^{12}$ no permite que los métodos que consiguen el acuerdo puedan ser utilizados de forma alternativa en el seguimiento de un paciente, por lo que el método seleccionado inicialmente debe seguir siendo utilizado en todo el proceso.

La valoración de la composición corporal, que forma parte de la evaluación del estado nutricional, va a adquirir un protagonismo creciente en los próximos años, favorecido por diversas situaciones sociosanitarias ${ }^{47,48}$, siendo las más relevantes por su impacto en la atención diaria el aumento de personas mayores de 65 años, el aumento de personas 
con sobrepeso, obesidad, anorexia o que simplemente quieren hacer dieta por motivos estéticos y el papel de la nutrición y del estado nutricional en la morbimortalidad por múltiples procesos crónicos a cualquier edad.

La anorexia es un problema relevante de salud en nuestra sociedad, habiendo incrementado no sólo su prevalencia, sino también el intervalo de edad de presentación. Pero la desnutrición también está comenzando a ser un problema de salud en edades avanzadas. El aumento en la esperanza de vida y el descenso de la natalidad conlleva un incremento progresivo en el número de personas mayores de 65 años, así como de su peso como colectivo frente al total de la población. La presencia de problemas físicos, psíquicos y sociales condicionados por el propio proceso de envejecimiento, por el cambio en la estructura familiar y por las barreras físicas de un urbanismo no pensado para ellas ${ }^{48}$ favorece la aparición de alteraciones nutricionales, que con relativa frecuencia son minusvaloradas por la presencia concomitante de múltiples patologías crónicas, sobre las que recae el grueso de la atención, situación que debe ir modificándose dada la importancia que tiene el estado nutricional en la morbimortalidad asociada a estos procesos.

También es un problema emergente y que condiciona cada vez mayor consumo de recursos el aumento del porcentaje de personas con sobrepeso y obesidad ${ }^{49}$, favorecido por la elevada disponibilidad de alimentos y por la sedentarización progresiva impuesta por los avances tecnológicos. La masa grasa sustituye a la masa muscular y para un mismo IMC los sujetos son más grasos, de forma que a determinados valores el IMC puede comenzar a ser un parámetro que no identifique adecuadamente a los sujetos con riesgo de complicaciones metabólicas o cardiovasculares y ser preciso, como ya se comienza a recomendar desde diversos consensos ${ }^{49,50}$ utilizar otras medidas antropométricas, como la circunferencia de la cintura, que en estos procesos es un factor de riesgo independiente, probablemente para cual- quier $\mathrm{IMC}^{50}$, y el \%GC ${ }^{15,49}$. Por ello, probablemente sea más eficiente realizar la valoración antropométrica del estado nutricional combinando todas estas medidas y la experiencia acumulada en torno a ellas y que los estudios que se diseñen para obtener tablas de referencia incorporen a las medidas clásicas (peso, talla, IMC) la circunferencia de la cintura, el porcentaje de grasa corporal y la circunferencia del brazo, planteamiento que ya se ve parcialmente recogido en algunos estudios ${ }^{7-9,51}$.

\section{AGRADECIMIENTOS}

Al Dr. Manuel de Oya Otero, catedrático del Departamento de Medicina de la Universidad Autónoma de Madrid y jefe de servicio de la Unidad de Lípidos de la Fundación Jiménez Díaz y al Dr. Agustín Gómez de la Cámara, de la Unidad de Investigación y Epidemiología Clínica del Hospital 12 de Octubre de Madrid, por sus aportaciones y comentarios. A la Sra. Elke Thomsen, por su colaboración en la traducción de artículos.

\section{BIBLIOGRAFÍA}

1. Alastrué A, Sitges A, Jaurrieta E, Sitges A. Valoración de los parámetros antropométricos en nuestra población. Med Clin (Barc) 1982; 10: 407-415.

2. Alastrué A, Sitges A, Jaurrieta E, Sitges A. Valoración antropométrica del estado de nutrición. Normas y criterios de desnutrición y obesidad. Med Clin (Barc) 1983; 16: 691-9.

3. Alastrué A, Rull M, Camps I, Salvá JA. Nuevas normas y consejos en la valoración de los parámetros antropométricos en nuestra población: índice adiposo muscular, índices ponderales y tablas de percentiles de los datos antropométricos útiles en una valoración nutricional. Med Clin (Barc) 1988; 91: 223-236

4. Ricart W, González-Huix F, Conde V y Grup per l'Evaluació de la Composició Corporal de la Població de Catalunya. Girona. Valoración del estado de nutrición a través de la determinación de los parámetros antropométricos: nuevas tablas en la población laboral de Cataluña. Med Clin (Barc) 1993; 100: 681-691. 
5. Aranceta J, Pérez C, Amela C, García R. Encuesta de Nutrición de la Comunidad de Madrid. Madrid: Dirección de Prevención y Promoción de la Salud; 1994.

6. Quiles Izquierdo J, Vioque J. Prevalencia de la obesidad en la Comunidad Valenciana. Med Clin (Barc) 1996; 106: 529-533.

7. Visser M, Langlois J, Guralnik JM, Cauley JA, Kronmal RA,Robbins J, et al. High body fatness, but not low fat-free mass, predicts disability in older men and women: the Cardiovascular Health Study. Am J Clin Nutr 1998; 68: 584-590.

8. Roubenoff R. Applications of bioelectrical impedance analysis for body composition to epidemiologic studies. Am J Clin Nutr 1996; 64: 459S-462S.

9. Kuczmarski RJ. Bioelectrical impedance analysis measurements as part of a national nutrition survey. Am J Clin Nutr 1996; 64: 453S-458S.

10. Pencharz PB, Azcue M. Use of bioelectrical impedance analysis measurements in the clinical management of malnutrition. Am J Clin Nutr 1996; 64: 485S-8S.

11. Vaisman N, Corey M, Rossi MF, Goldberg E, Pencharz P. Changes in body composition during refeeding of patients with anorexia nervosa. J Pediatr 1988; 113: 925-9.

12. Valtueña Martínez S, Arija Aval V, Salas-Salvadó J. Estado actual de los métodos de evaluación de la composición corporal: descripción, reproducibilidad, precisión, ámbitos de aplicación, seguridad, coste y perspectivas de futuro. Med Clin (Barc) 1996; 106: 624-635.

13. Bretón Lesmes I, Cuerda Compés M. ${ }^{\mathrm{a}} \mathrm{C}$, Camblor Alvarez M, García Peris P. Técnicas de composición corporal en el estudio de la obesidad. En: Moreno Esteban B, Monereo Megías S, Álvarez Hernández J. Obesidad. La epidemia del siglo XXI. Ediciones Díaz de Santos; 2000.p. 169-190.

14. Lukaski HC. Methods for the assessment of human body composition: traditional and new. Am J Clin Nutr 1987; 46: 537-556.

15. López Calbet JA, Armengol Ramos O, Chavarren Cabrero J, Dorado García C. Una ecuación antropométrica para la determinación del porcentaje de grasa corporal en varones jóvenes de la población canaria. Med Clin (Barc) 1997; 108: 207-213.

16. García-Lorda P, Salas-Salvadó J. Evaluación de la composición corporal en el paciente obeso. Medicina Integral 1999; 6: 262-271.
17. Siri WE. Body composition from fluid spaces and density: analysis of methods. En: Brozeck J, Henschel A eds. Techniques for measuring body composition. Washington DC: National Academy of Sciences. Natural Resourcer Council, 1961: 223-244.

18. Brozeck J, Grande F, Anderson JT, Keys A. Densitometric analysis of body composition: revision of some quantitative assumptions. Ann N Y Acad Sci 1963; 110: 113-140.

19. Deurenberg P, Wetstrate JA, Seidell JC. Body mass index as a measure of body fatness: age- and sex- specific prediction formulas. Br J Nutr 1991; 65: $105-114$

20. Lean MEJ, Han TS, Deurenberg P. Predicting body composition by densitometry from simple anthropometric measurements. Am J Clin Nutr 1996; 63: 4-14.

21. Núñez C, Carvajal A, Turrero E, Moreiras O Contribución al estudio de la composición corporal de un grupo de mujeres jóvenes mediante análisis de impedancia bioeléctrica. Nutr Hosp. 1994; 9: 262-267.

22. Durnin JVGA, Womersley J. Body fat assessed from total body density and its estimation from skinfold thickness: measurements on 481 men and women aged from 16 to 72 years. Br J Nutr 1974; 32: 77-97.

23. Sociedad Española para el Estudio de la Obesidad (SEEDO). Consenso español 1995 para la evaluación de la obesidad y para la realización de estudios epidemiológicos. Med Clin (Barc) 1996; 107: 782-787.

24. Prieto L, Lamarca R, Casado A. La evaluación de la fiabilidad en las observaciones clínicas: el coeficiente de correlación intraclase. Med Clin (Barc) 1998; 110: 142-145.

25. Bland JM, Altman DG. Statistical methods for assessing agreement between two methods of clinical measurement. Lancet 1986; 1: 307-310.

26. Lohman TG. Skinfold and body density and their relation to body fatness: a review. Hum Biol 1981; 53: 181-225.

27. Scherf J, Franklin BA, Iucas CP. Validity of skinfold thickness measures of formerly obese adults. Am J Clin Nutr 1986; 43: 128-135.

28. Roche AF. Grading body fatness limited anthrpometric data. Am J Clin Nutr 1981; 34: 2521-2529. 
29. Brozeck J, Keys A. The evaluation of leanness-fatnnes in man: norms and interrelatioships. Br J Nutr 1951; 5: 194-206.

30. Bray GA, Greenway FL, Molitch ME. Use of anthropometric measures to assess weigth loss. Am J Clin Nutr 1978; 31: 769-773.

31. Valero MA, León-Sánz M, Gómez I, Martínez G, Hawkins F. Comparación entre absorciometría de doble fotón (DEXA), impedancia y antropometría en el estudio de la composición corporal en personas obesas. Nutr Hosp 1994; 9: 12-17.

32. Rush EC, Plank LD, Laulu MS, Robinson SM. Prediction of percentage body fat from anthropometric measurements: comparison of New Zealand Eurpoean and Polynesian young women. Am J Clin Nutr 1997; 66: 2-7.

33. Alastrué Vidal A. Antropometría y obesidad. Med Clin (Barc) 1994; 102: 16-19.

34. Gómez de la Cámara A, Cruz Martos E, de la Cruz Bértolo J, Landa Goñi J, Guillomía Contreras S, Aurrecoechea R. Análisis de la fiabilidad de tres dosímetros portátiles de glucemia. Comparación de diferentes métodos para el estudio de la fiabilidad de las observaciones clínicas. Med Clin (Barc) 1997; 108: 410-3.

35. Fermanian J. Mesure de l'accord entre deux juges: cas quantitatif. Rev Epidém Santé Publ 1984; 32: 408-413.

36. McNeill, Fowler PA, Maughan RJ, McGaw BA, Fuller MF, Gvozdanovic D et al. Body fat in lean and overweigth women estimated by six methods. Br J Nutr 1991; 65: 95-103.

37. Jebb SA, Cole TJ, Doman D, Murgatroyd PR, Prentice AM. Evaluation of the novel Tanita body-fat analyser to measure body composition by comparison with a four-compartment model. Br J Nutr 2000; 83: 115-122.

38. Wong WW, Stuff JE, Butte NF, O'Brian Smith E, Ellis J. Estimating body fat in African American and white adolescent grils: a comparison of skinfold-thickness equations with a 4-compartment model. Am J Clin Nutr 2000; 72: 348-354.

39. Gutin B, Litaker M, Islam S, Manos T, Smith C, Treiber F. Body-composition measurement in 9-11-y-old children by dual-energy X.ray absorptiometry, skinfold-thickness measurements, and bioimpedance analysis. Am J Clin Nutr 1996; 63: 287-292.

40. Houtkooper LB, Lohman TG, Going SB, Howell WH. Why bioelectrical impedance analysis should be used for estimating adiposity. Am J Clin Nutr 1996; 64: 436S-448S.
41. Brodie D, Moscrip V, Hutcheon R. Body Composition Measurement: A Review of Hydrodensitometry, Antrhopometry, and Impedance Methods. Nutrition 1998; 14: 296-310.

42. Loy SF, Likesn BA, Andrews PM, and cols. Easy grip on body composition measurements. ACSM's Health Fit J 1998; 2: 16-19.

43. Gibson AL, Heyward VH, Mermier CM. Predictive accuracy of Omron body logic analyzer in estimating relative body fat of adults. Int J Sport Nutr Exerc Metab 2000; 10: 216-227.

44. National Institutes of Health Technology Assessment. Bioelectrical impedance analysis in body composition measurement: National Institutes of Health Technology Assessment Conference Statement. Am J Clin Nutr 1996; 64: 524S-532S.

45. Fernández López JA, Alemany M, Remesar X. Discordancias en la aplicación de sistemas comerciales de medida de la grasa corporal. Endocrinología y Nutrición 2000; 47: 73-76.

46. Yannakuolia M, Keramopoulos A, Tsakalakos N, Matalas AL. Body composition in dancers: the bioelectrical impedance method. Med Sci Sports Exerc 2000; 32: 228-236.

47. Martínez Valls JF. Diagnóstico y valoración del estado nutricional. Medicine 7. a serie, 1999; 110 : 5125-5135.

48. Espinosa Almendro JM. El anciano en atención primaria. Aten Primaria 2000; 26: 515-516.

49. Sociedad Española para el Estudio de la Obesidad (SEEDO). Conferencia de consenso. Consenso SEEDO'2000 para la evaluación del sobrepeso y la obesidad y el establecimiento de criterios de intervención terapéutica. Med Clin (Barc) 2000; 115: $587-597$.

50. Expert Panel on the Identification, Evaluation and Treatment of Overweight in Adults. Clinical Guidelines on the Identification, Evaluation, and Treatment of Overweight and Obesity in Adults: Executive Summary. NHLBI Clinical Guidelines Executive Summary. Am J Clin Nutr 1998; 68: 899-917.

51. Gallagher D, Heymsfield SB, Heo M, Jebb SA, Murgatroyd PR, Sakamoto Y. Healthy percentage body fat ranges: an approach for developing guidelines on body mass index. Am J Clin Nutr 2000; 72: 694-701. 